\title{
MICROFABRICATION AND PACKAGING OF A RUBIDIUM VAPOR CELL AS A PLASMA LIGHT SOURCE FOR MEMS ATOMIC CLOCKS
}

\author{
V. Venkatraman ${ }^{1}$, Y. Petremand ${ }^{2}$, C. Affolderbach ${ }^{3}$, G. Mileti $^{3}$, N. de Rooij ${ }^{2}$, and H. Shea ${ }^{1}$ \\ ${ }^{1}$ LMTS, Ecole Polytechnique Federale de Lausanne (EPFL), Switzerland \\ ${ }^{2}$ SAMLAB, Ecole Polytechnique Federale de Lausanne (EPFL), Switzerland \\ ${ }^{3}$ Laboratoire Temps-Fréquence (LTF), University of Neuchatel, Switzerland
}

\begin{abstract}
We report on the micro-fabrication and characterization of a chip-scale plasma light source based on a Rubidium $(\mathrm{Rb})$ vapor cell. The $\mathrm{Rb}$ plasma light source is intended for use as an integrated optical pump-light source in miniature double-resonance $\mathrm{Rb}$ atomic clocks $[1,5]$. The RF plasma is capacitively coupled using external electrodes, and the light source is impedance matched to the source for frequencies between 1 and $36 \mathrm{MHz}$. Rb vapor cells have been previously developed as reference cells for atomic clocks but not as light sources. This is the first reported $\mathrm{Rb}$ plasma emitted from a chip-scale device. Stable light emission is observed for over 18 days.
\end{abstract}

\section{KEYWORDS}

Plasma light source, rubidium, chip-scale atomic clocks, anodic bonding

\section{INTRODUCTION}

Miniaturizing atomic clocks to reach sub- $\mathrm{cm}^{3}$ volumes for applications including GPS receivers and communication systems is a very active field [1]. Compact $\left(\sim 10 \mathrm{~cm}^{3}\right)$ commercial Rubidium atomic clocks rely on $\mathrm{Rb}$ plasma discharge lamps as the light source due to the lamp's intrinsically correct frequency $(\mathrm{Rb}$ lines), technical simplicity, very slow frequency drift (aging), and long lifetime.

Microfabricating hermetically sealed $\mathrm{mm}^{3} \mathrm{Rb}$ cells has been a challenge, with several groups reporting different bonding techniques compatible with $\mathrm{Rb}$ filling, for instance anodic bonding [2] or soldering [3]. Such cells have been used as the reference cell in miniaturized atomic frequency standards (atomic clocks), but not as light sources - in part due to the additional complexity of igniting a stable plasma. While first studies on micro-cell plasma light sources reported on low-power rare-gas discharges, no Rb light was reported [10, 11].

Current state-of-the-art miniature atomic clocks use a laser diode (VCSEL) as the light source for atomic resonance [4] because of their easy integration, good power efficiency, planarity and compact dimensions $\left(\sim \mathrm{mm}^{2}\right)$. However, the VCSEL approach requires sophisticated control electronics for laser frequency stabilization [5], critical temperature control requirements and complicated setup procedures [1]. The goal of our work is to develop a microfabricated planar $\mathrm{Rb}$ plasma light source (as shown in operation in Figure 1) as an alternative and innovative design for MEMS atomic clocks (as shown schematically in Figure 2).

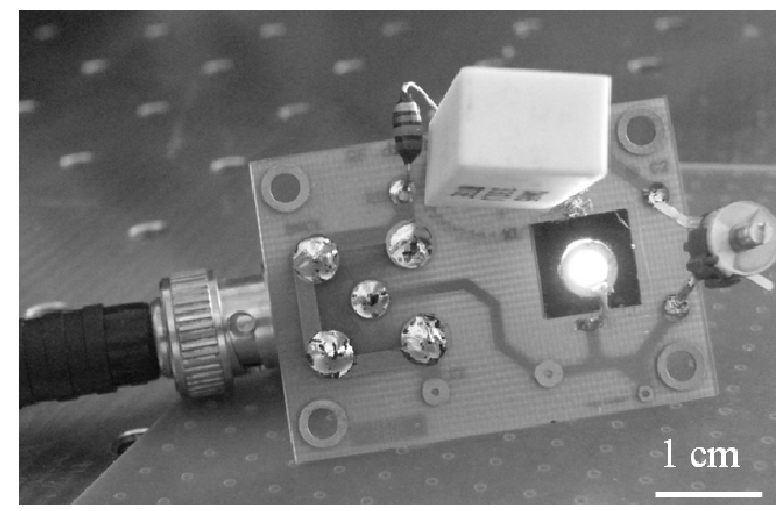

Figure 1: Rb plasma cell with the impedance matching circuit. The light emitted from the center of the cell indicates the ignited plasma

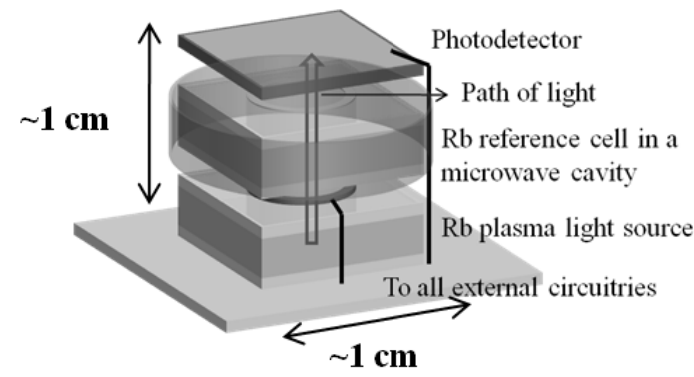

Figure 2: Schematic design of a chip-scale double-resonance atomic clock into which the Rb plasma light source will be integrated

\section{Double-resonance Rb atomic clock}

The fundamental concept behind timekeeping in atomic clocks is to exploit an atomic resonance as reference for stabilizing the frequency of a quartz oscillator. In a $\mathrm{Rb}$ atomic clock [5], this resonance is the "clock" transition between the two ground-state hyperfine levels of ${ }^{87} \mathrm{Rb}$, at a microwave frequency near $6.83 \mathrm{GHz}\left(5^{2} \mathrm{~S}_{1 / 2}\right.$ state, $\left|\mathrm{F}=2, \mathrm{~m}_{\mathrm{F}}=0\right\rangle$ to $\left.\left|\mathrm{F}=1, \mathrm{~m}_{\mathrm{F}}=0\right\rangle\right)$, detected on atoms maintained in a small $\mathrm{Rb}$ reference cell. In a double-resonance $\mathrm{Rb}$ clock, pump light from a lamp or laser resonant with the Rb D1 or D2 line (794.8 $\mathrm{nm}$ or $780.2 \mathrm{~nm}$, respectively) irradiates the cell and creates a ground-state polarization in the atoms. The clock transition is then detected by applying a microwave field to the atoms via a microwave-cavity placed around the cell, and the resonance manifests itself 
as a narrow dip in pump-light power transmitted through the cell. The frequency of a voltage-controlled quartz oscillator is locked to this dip using phase-sensitive detection.

Our envisaged double-resonance $\mathrm{Rb}$ atomic clock consists of a micro-fabricated $\mathrm{Rb}$ (natural) plasma light source, a micro-fabricated $\mathrm{Rb}$ reference cell which is enclosed by a microwave cavity, and a photodetector (see Figure 2). The Rb plasma light source intrinsically emits at the correct wavelengths, Rb D1 and D2 lines, as required in a $\mathrm{Rb}$ clock.

\section{Rb Plasma Glow Discharge}

Igniting the plasma in a $\mathrm{Rb}$ cell requires ionizing the $\mathrm{Rb}$ atoms which are in the vapor phase. When a sufficiently strong electric field is applied across a gas, it ionizes the gas and accelerates the positive ions and free electrons towards the opposite electrodes. During this process, if the electric field is strong enough, the ions and electrons collide with the neutral vapor atoms generating free electrons and positive ions, through impact ionization. This becomes an avalanche process, eventually initiating an electrical breakdown leading to several possible types of gas discharge [6].

The discharge of interest to us is the Rb plasma glow discharge which includes the Rb lines - D1 and D2 that are used to maintain optical resonance in an atomic clock. This light source is aimed to be integrated into portable chip-scale atomic clocks, so it must consume very little electrical power. Because the $\mathrm{Rb}$ vapor density is very low at room temperature $\left(\sim 10^{-6}\right.$ mbar at $\left.30^{\circ} \mathrm{C}\right)$, an extremely high field strength would be needed to initiate electrical breakdown (Paschen curve) [7], and the $\mathrm{Rb}$ light intensity would be low.

Two steps are carried out to address these two problems: (1) Increasing the temperature of the cell increases the $\mathrm{Rb}$ vapor density and (2) adding a low pressure starter gas to the $\mathrm{Rb}$ cell to increase the density of gas atoms in the cell. The gas needs to have a low ionization potential but higher than that of $\mathrm{Rb}$ (First Ionization energy, IE1: $4.17 \mathrm{eV}$ ), in order to ionize $\mathrm{Rb}$ easier than the buffer gas after plasma ignition. $\mathrm{Rb}$ is highly reactive with several gases, especially oxygen. Argon, an inert gas, which is monoatomic (hence has a relatively smaller diameter and higher mean free path among noble gases), having an ionization potential of $15.75 \mathrm{eV}$ [8] is a good choice for this purpose.

\section{DEVICE DEVELOPMENT \\ Design and fabrication}

The design approach chosen for the $\mathrm{Rb}$ plasma light source is the electrodeless capacitively coupled plasma (CCP). Capacitive coupling produces stronger field lines in a planar cell cavity to ignite a plasma than the more widely used inductive coupling of plasma which is more efficient for spherical cells.

The microfabricated device $\left(1 \times 1 \times 0.3 \mathrm{~cm}^{3}\right)$, consists of a stack of three layers: Pyrex $(500 \mu \mathrm{m})$, Silicon $(2$ $\mathrm{mm})$, Pyrex $(500 \mu \mathrm{m})$ which enclose a $\Phi=5 \mathrm{~mm}, h=2$ $\mathrm{mm}$ cylindrical cavity in which few $\mu \mathrm{l}$ of Rubidium and 70 mbar of Argon are hermetically sealed by a two-step anodic bonding process (Figure 3 ). First, the cavity is created by DRIE etching a through-hole in the silicon wafer. Then, the etched silicon wafer is anodically bonded to the bottom Pyrex layer. After this first bonding step, the natural $\mathrm{Rb}$ is dispensed into the cavity under vacuum by an atomic jet, 70 mbar of Argon is introduced in the chamber, and the top Pyrex layer is anodically bonded to the bottom layers to create the hermetically sealed $\mathrm{Rb}$ mini-cell [12].

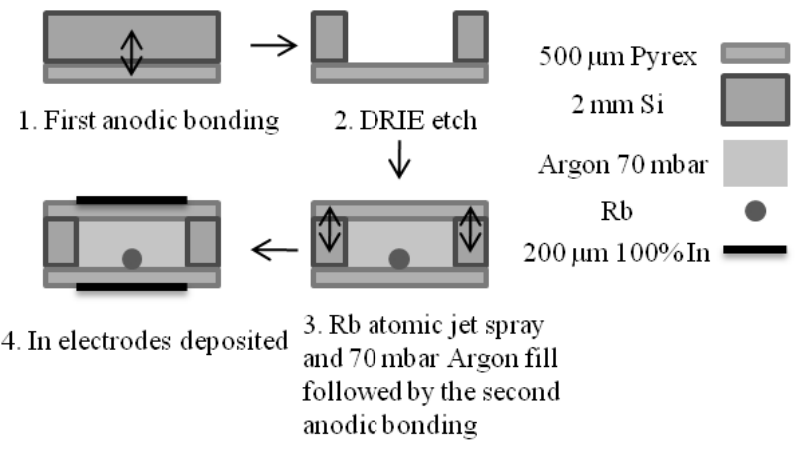

Figure 3: Microfabrication process of the Rb plasma light source

A $200 \mu \mathrm{m}$ thick $100 \%$ Indium solder is deposited on top of both sides of the Pyrex layers (i.e., outside the cell) to act as capacitive parallel electrodes to capacitively couple a plasma in the cell. $100 \%$ Indium wets glass well and is hence a good choice for deposition on the Pyrex walls. The $100 \%$ Indium electrodes are deposited by placing and aligning Indium pre-form rings on top of Pyrex layers above the cavity and affixed using a soldering iron. These external electrodes avoid electrode corrosion and might allow for lower power consumption when compared to the more traditional internal electrode designs [9].

\section{Impedance Matching and RF Electronics}

In order to create the breakdown voltage across the cell with the lowest input electrical power, the cell needs to be impedance matched with the source at $50 \Omega$. The microfabricated $\mathrm{Rb}$ vapor cell has an intrinsic capacitance of $\sim 0.4 \mathrm{pF}$ at low frequencies $(<10 \mathrm{MHz})$ and up to $\sim 1.6 \mathrm{pF}$ at higher frequencies $(<36 \mathrm{MHz}$ ), having a reactance of $\sim 86.5 \mathrm{k} \Omega$ at $4.6 \mathrm{MHz}$. It is electrically connected to specific LCR components to build a zero reactive and $50 \Omega$ resistive load $-(50+\mathrm{j}(0)$ $\Omega)$. This maximizes the power transfer from the input to the cell and hence lowers the overall power consumption of the circuit. Figure 4 shows schematically the RF plasma ignition circuit connected to the impedance matched load constituting the light source. 


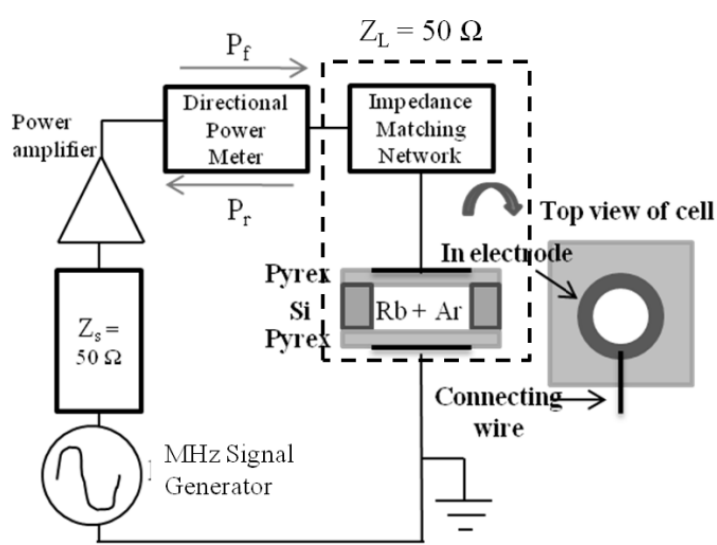

Figure 4: Plasma ignition schematic circuit with crosssectional views of the cell

\section{RESULTS}

The plasma light source was impedance matched and tested at several frequency points over the $1-36 \mathrm{MHz}$ range and results obtained when tested at $4.6 \mathrm{MHz}$ is reported in this article.

The light source was characterized in terms of the following: (1) total output optical power emitted by the light source, (2) total electrical power consumed and (3) the stability of the $\mathrm{Rb}$ lines with time.

The output optical spectrum of the plasma glow discharge was measured using a spectrometer where strong Rb lines - D1 (794.8 nm) and D2 (780.2 nm) at temperatures above $90{ }^{\circ} \mathrm{C}$, the working temperature range, was observed. Figure 5 shows the optical spectrum of the light source at different temperatures from $35{ }^{\circ} \mathrm{C}$ to $151{ }^{\circ} \mathrm{C}$. A rapid rise in intensity of the $\mathrm{Rb}$ lines with increase in cell temperature is observed as expected as the $\mathrm{Rb}$ vapor pressure increases rapidly inside the cell with temperature.

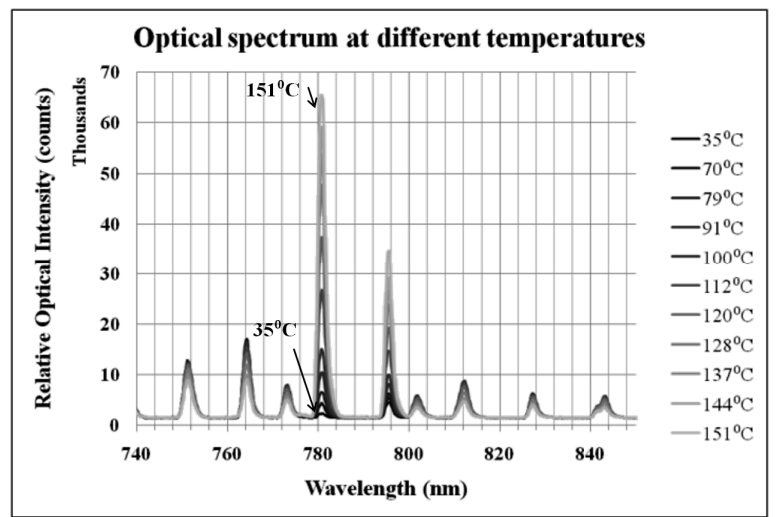

Figure 5: Output optical spectrum of the $R b$ plasma light source showing both $\mathrm{Rb} D 1$ and D2 lines at $151^{\circ} \mathrm{C}$

At 4.6 MHz, $100 \mu \mathrm{W}$ of total optical power was measured using an optical power meter when the electrical power consumption by the light source was calculated to be less than $5 \mathrm{~mW}$ while the total consumption by the RF circuit was $620 \mathrm{~mW}$ (the matching circuit dissipates most of the RF power).

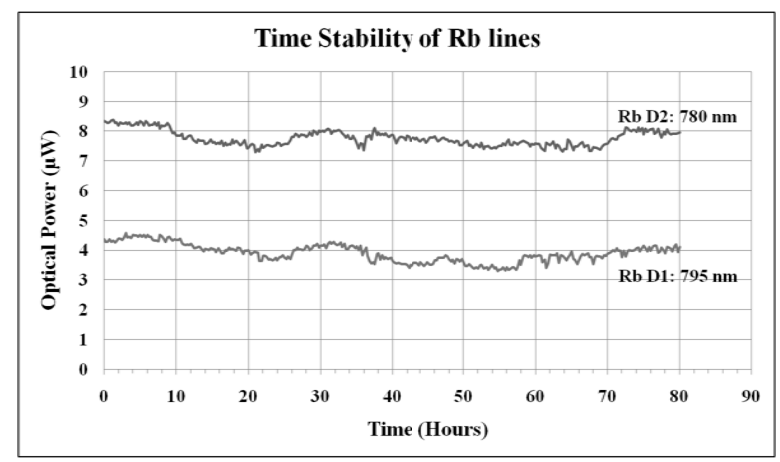

Figure 6: Both Rb D1 and D2 lines were found to emit stable power over time (measured at $100^{\circ} \mathrm{C}$ )

The stability of the output optical power of the $R b$ lines was observed over time at the working temperature range of the plasma light source $\left(>90{ }^{\circ} \mathrm{C}\right)$ by measuring short-term ( $\sim$ seconds) and long term ( $\sim$ hours $)$ variation in optical intensity. The average short term fluctuations in optical intensity of the $\mathrm{Rb}$ lines was found to be less than $0.1 \%$ and the long-term variations were found to be less than 5\% (shown in Figure 6). The primary cause of the long-term variations was the observed drifts in resistance $(\sim 2-3 \%)$ of the electrical components of the impedance matching network with time. The long-term variations can be limited to less than $0.01 \%$ using a PID feedback loop by controlling the input electrical power to maintain steady output light.

\section{CONCLUSIONS}

A stable and low-power chip-scale Rb optical pump with potential for wafer-scale fabrication has been demonstrated for MEMS atomic clocks, emitting several $\mu \mathrm{W}$ of optical power at the 780.2 and $794.8 \mathrm{~nm} \mathrm{Rb}$ lines from a $0.3 \mathrm{~cm}^{3}$ chip stack. A successful $\mathrm{Rb}$ hermetic sealing technique through standard microfabrication processes has been demonstrated. An important factor in the longevity is the external electrodes which cannot be eroded by the plasma. The light source could also be readily adapted for other applications including atomic magnetometers and gyroscopes.

In the present design most of the electrical power is dissipated by the electrical components. Current research is towards minimizing these losses and increasing the efficiency of power transfer to the cell.

\section{ACKNOWLEDGMENTS}

This work was supported by the Swiss National Science Foundation, Sinergia grant CRSI20-122693/1.

\section{REFERENCES}

[1] S. Knappe, "MEMS Atomic Clocks", Comprehensive Microsystems, Vol. 3, pp. 571-612, 
2007.

[2] A. Douahi, L. Nieradko, J. C. Beugnot, J. Dziuban, H. Maillote, S. Guerandel, M. Moraja, C. Gorecki, V. Giordano : "Vapour microcell for chip scale atomic frequency standard", Electronics Letters, Vol. 43, pp. 279-280, 2007.

[3] F. Vecchio, V. Venkatraman, H. Shea, T. Maedar, P. Ryser, "Dispensing and Hermetic Sealing $\mathrm{Rb}$ in a Miniature Reference Cell", Sensors \& Actuators: A. Physical, [In press]

[4] S. Knappe, V. Shah, P. Schwindt, L. Holberg, J. Kitching, "A microfabricated atomic clock", Appl. Phys. Lett., Vol. 85, pp. 1460-1462, 2004.

[5] J. Camparo, "The rubidium atomic clock and basic research", Phys. Today, Vol. 60, pp. 33-39, 2007.

[6] J. H. Gross, "Mass Spectrometry: A Textbook". $4^{\text {th }}$ edition, Berlin: Springer, 2004.

[7] F. Paschen, "Über die zum Funkenübergang in Luft, Wasserstoff and Kohlensäure bei verschiedenen Drücken erforderliche Potentialdifferenz", Weid. Annalen der Physick, Vol.37, pp. 69-75, 1889.

[8] E. Nasser, "Fundamentals of Gaseous Ionization and Plasma Electronics", Wiley-Interscience, 1971.
[9] D. O. Wharmby, "Electrodeless lamps for lighting: a review", IEE-Proceedings, Vol. 140, pp. 465-473, 1993.

[10]H. Guo, A. Lal, "Self-Powered Photon Source", IEEE Proc. of the $12^{\text {th }}$ Int. Conference on Solid State Sensors, Actuators and Microsystems, Transducers '03, Boston, U.S.A, 2003, pp. 1474-1477.

[11]S. Wang, L. Lin, H. Guo, “Analysis and design of a micromachined $\mathrm{Rb}-85$ filter in passive Rubidium atomic clock", Proc. IEEE Int. Conference on Nano/Micro Engineered and Molecular Systems, Shenzen, China, 2009, p. 911-914.

[12]J. DiFrancesco, F. Gruet, C. Schori, C. Affolderbach, R. Matthey, G. Mileti, Y. Salvadé, Y. Petremand, N. De Rooij, "Evaluation of the frequency stability of a VCSEL locked to a microfabricated Rubidium vapour cell", Proc. SPIE, Vol. 7720, pp. 77201T, 2010.

\section{CONTACT}

* V. Venkatraman, Tel: +41-32-729-5259; E-mail: vinu.venkat@epfl.ch 